\title{
Editorial for SM 160 - Design and Implementation of Mobile Smart Objects Special Issue
}

\author{
Pietro Manzoni ${ }^{1} \cdot$ Claudio Palazzi ${ }^{2}$
}

Published online: 16 April 2016

(C) Springer Science+Business Media New York 2016

\section{Editorial:}

This special issue features six selected high quality papers from SMARTOBJECTS 2015, a Workshop on experiences with the design and implementation of smart objects, held of September 7th, 2015 in Paris, FRANCE. It focuses on experiences with the design, implementation, deployment, operation and evaluation of novel communication approaches and systems for smart objects in the emerging cooperative environments.

In the first article, "An NFC Anti-Counterfeiting Framework for ID Verification and Image Protection", Wei Hsun Lee, Chien Ming Chou, and Szu Wen Wang propose an NFC anti-counterfeiting framework with a two-layered digital image protection mechanism. In this system, an application certificate signed by the service provider and image metadata are hidden in the personal digital image using digital watermarking, and the image metadata is protected by the secure element in the NFC device. Several procedures are designed to ensure the originality of the photo and signature images, so that the process of digital image authentication is as secure and practical as using printed images on a plastic card.

Pietro Manzoni

pmanzoni@disca.upv.es

Universitat Politècnica de Valéncia, Valencia, Spain

2 University of Padua, Padova, Italy
The second article titled "The Coordinated Vehicle Recovery Mechanism in City Environments", by GwoJiun Horng, presents the design and analysis of an innovative, optimized and coordinated vehicle recovery network system. This system safeguards private property for all citizens, significantly enhances an existing vehicle recovery and anti-theft system, and improves the success rate for vehicle recovery. This study is not only applicable to a single vehicle but also considers the entire vehicle anti-theft communication network and connects all individual vehicles to an innovative, optimized and coordinated vehicle recovery network.

The next article "A Network Coverage Algorithm for Message Broadcast in Vehicular Networks", by Armir Bujari, proposes an enhancement of an existent optimal message propagation scheme proposed in the context of one dimensional vehicular networks, namely Fast-Broadcast. This proposal generalizes the former with the objective of guiding message dissemination to an entire area of interest in generic road topologies. The proposed scheme exploits bloom filter properties to further advance message propagation into the network.

The fourth article is titled "Wearable HUD for Ecological Field Research Applications: A Development Experience on the Design of a Wearable Mobile System". Its authors, Saul Delabrida, Thiago D'Angelo, Ricardo Augusto Rabelo Oliveira, and Antônio Alfredo F. Loureiro, overview wearable architectures found in the literature and present a novel wearable device for monitoring ecological environments. The wearable includes a Head-UP Display (HUD) assembled with Google Card- 
board API and sensors connected to a development board. This device provides several functionalities such as distance measurement to objects and weather conditions monitoring. Camera and green lasers combined with a digital image-processing algorithm are used to measure the distance to objects.

The fifth article, "Sensing Pollution on Online Social Networks: A Transportation Perspective", by Rita Tse, Yubin Xiao, Giovanni Pau, Serge Fdida, Marco Roccetti, and Gustavo Marfia, verifies the feasibility of sensing air pollution from social networks and of integrating such information with real sensors feeds, unveiling how people advertise such phenomenon, acting themselves as smart objects, and how online posts relate to true pollution levels. This work explores a new dimension in pollution sensing for the benefit of environmental and transportation research in future smart cities, confronting over 1,500,000 posts and pollution readings obtained from governmental on-the-field sensors over a 1-year span.

In the last article titled "A Mission-Oriented Coordination Framework for Teams of Mobile Aerial and Terrestrial Smart Objects", the authors (Pasquale Pace, Gianluca Aloi, Giuseppe Caliciuri, and Giancarlo Fortino) propose and investigate a novel framework to support both the management and the collaboration of Mobile Smart Objects (MSOs) considered as terrestrial and aerial drones (i.e., UAVs, UGVs). MSOs are equipped with embedded sensors and/or actuators and can move autonomously always remaining connected, accessible and controllable. The proposed framework allows the programming and management of smart drones and the coordination of teams of drones according to a mission-oriented paradigm.

The guest editors are thankful to our reviewers for their effort in reviewing the manuscripts. We also thank the Editor-in-Chief, Dr. Imrich Chlamtac for his supportive guidance during the entire process.

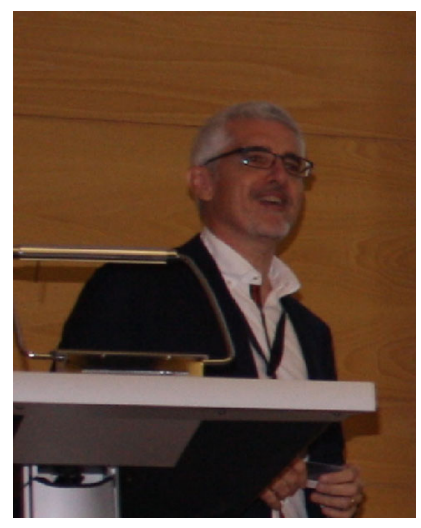

Prof. Pietro Manzoni received the MS degree in computer science from the "Università degli Studi" of Milan, Italy, in 1989, and the PhD degree in computer science from the "Politecnico di Milano", Italy, in 1995 . He is currently a full professor of computer science at the "Universitat Politècnica de València", Spain. His research activity is related to mobile wireless data systems design, modeling, and implementation, particularly oriented to Intelligent Transport Systems and Smart Cities. He published more than 200 scientific papers; his H-index is 28 according to Google Scholar. He has been actively involved in the organization or in the technical committee of various scientific conference and journals. He collaborates with various international academic and industrial research centers. He is a member of the IEEE.

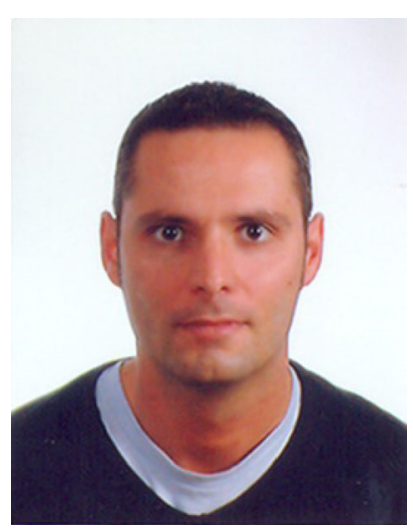

Prof. Claudio Palazzi is an Associate Professor of Computer Science at the University of Padua. He received his M.S. degree in Computer Science from UCLA in 2005, his Ph.D. degree in Computer Science from University of Bologna in 2006, and his Ph.D. degree in Computer Science from UCLA in 2007. His research interests are primarily focused on the design and analysis of communication protocols for wired/wireless networks, with an emphasis on mobile applications and multimedia entertainment. He is active in various technical program committees of the most prominent international conferences and is author of more than 120 papers, published in international conference proceedings, books, and journals. He was TPC Chair of IEEE CCNC 2016 and General Chair of IFIP/IEEE Wireless Days 2010. He currently is General Chair of MobiSys/DroNet and MobiCom/SMARTOBJECTS, and associate editor of the Elsevier Computer Networks journal. 